\title{
Studying Effects and Process in Psychotherapy for Personality Disorders
}

\author{
Franz Caspar \\ Clinical Psychology and Psychotherapy, University of Bern, Bern, Switzerland
}

\section{Keywords}

Psychotherapy for personality disorders - Psychotherapy research · Heterogeneity · Ego-syntony · Ambivalent motivation

\begin{abstract}
This presentation gives an overview of the methods used for research on the process and outcome of psychotherapy. Possibilities as well as difficulties will be discussed, such as the conflict between internal and external validity and standardized versus individualized procedures as some of the issues deserving particular attention for research on psychotherapy for personality disorder patients. It is argued that good psychotherapy research is also good psychotherapy research for personality disorders, with heterogeneity, egosyntony, and ambivalent motivation needing special attention. Adaptations of and alternatives for randomized clinical trials will be discussed.

(c) 2018 S. Karger AG, Basel
\end{abstract}

\section{Preliminary Comment}

Although at some point a particular perspective is taken, this article is mainly seen as an attempt to give a general introduction and overview rather than propagating a specific approach.

\section{KARGER}

(๑) 2018 S. Karger AG, Basel

E-Mail karger@karger.com

www.karger.com/psp

\section{Randomized Controlled Trials}

It is hard to write about psychotherapy research without referring to randomized controlled trials (RCTs), which are still seen as the "gold standard" for treatment research in general. While RCT designs are still the most conclusive as far as causal argumentation is concerned, they have disadvantages which have been discussed at large [1]. RCTs are not always needed in research on the effects of psychotherapy, at least not with the common manualization of procedures and homogenization of patients.

Historically, an effort was made by the American Psychological Association [2] to give equal chances to psychotherapy in competition with medication, by establishing psychotherapy as a standard product with proven efficacy. Complications such as giving weight to a therapist's characteristics were in the way of such an effort, just as it would be detrimental to advertise psychoactive medication if one were of the opinion that all depends on the personality of the pharmacist who delivers the drug and the relationship to the prescribing doctor. Since the acknowledgment of differences between therapists has not been encouraged, this has led to the fact that therapist effects are drastically underresearched [3].

The main problems of RCTs to be mentioned here, with particular relevance to the treatment of personality disorders, are:
Prof. Dr. Franz Caspar

E-Mail caspar@psy.unibe.ch 
1. The usual selection/homogenization of patients limits the external validity. The question always is: do the findings actually apply to the patient whom I want to treat? For personality disorders, comorbidity (which is usually, although not always, excluded) is a big issue

2. The effects reported from RCTs are usually achieved by therapists who are highly trained and supervised in their performance for a particular approach, whereas the training of therapists who follow the approach in regular practice is usually not as comprehensive. This also limits external validity/applicability of the findings

3. Related to the last point: in common practice, it is unrealistic for most therapists to specialize on one or two personality disorders; as a consequence, see point 2

For the most frequent disorders, there are several manualized therapeutic approaches, but there is still a big majority of patient groups for which there is no approach based on RCT evidence. If one considers comorbidity, for example, a depressed patient with a dependent personality background may require an at least partially different approach than a depressed patient with a narcissistic background; thus, evidence also shrinks for the major diagnostic groups. How satisfactory is it to tell a high percentage of patients that "psychotherapy is wonderful and we have a great number of wonderful evidence-based approaches, but unfortunately you have the wrong disorder?" As it takes enormous time and resources to bring a therapeutic approach through all steps of qualification (www.cochrane.org/), it is completely unrealistic that we will ever have empirically supported treatments for most patient groups - at least in line with the commonly held restrictive rules.

While there are several good reasons to be critical of RCT evidence, it is not the only but still the most conclusive methodology when it comes to causal argumentation. There are, however, two lines of argumentation to deal with this situation. First, we do not need RCTs for proving effects, let alone for all research questions. Gordon and Jill [4] wrote a half-serious report on an attempted meta-analysis for the reputable British Medical Journal (BMJ): before the use of parachutes can be recommended, it has to be proven that it actually makes a difference whether one uses them or not. They made a comprehensive search for RCTs related to that matter and found none. The use of parachutes can therefore, so the conclusion, not be recommended. The point here is, of course, that what Grawe (Smith and Grawe [5]) designated a "selection from natural variation," that is, the analysis of cases in which a particular constellation occurred naturally (without randomization), is absolutely sufficient: one has to study the cases in which a person jumped off an airplane and the parachute did not open for some non-experimental reason. Although there are a few reports of survivors, the consequences are absolutely clear, and nobody would reasonably ask for a randomized experiment.

Needless to say that in psychotherapy, much is not as clear (parachute opened yes/no; person survived yes/no), but there are many instances in which an experiment would be difficult, yet non-experimental findings might convince nevertheless; for example, for the question: "what happens in therapies which are successful in spite of a bad therapeutic relationship?"

A second point to be mentioned here is the following: it is obvious that it must be known what the therapeutic procedure was which led to the reported effects. The common way to achieve this is to develop a manual, to train and supervise therapists, and then to check by adherence ratings whether they actually did what they were supposed to do. To see this as the only possible way of knowing what the procedure was is, however, a mistake of mixing up means with the goal. The mistake is particularly serious, as the requirement of describing a procedure in detail, algorithmically, restricts the possibility of describing an approach (or condition in an RCT) in a rather flexible, heuristic way, which would be much more in line with the need to adapt to individual patients in normal practice, in particular with notoriously heterogeneous personality disorders and comorbidity.

How would one go about an RCT with a heuristic procedure? The heuristics (for example to develop an individualized procedure in one condition based on one kind of case formulation, and on another kind in the other condition) have to be clearly defined and they need to be distinctive between the conditions. The procedure developed on the basis of these heuristics has also to be trained and supervised, but can be very flexible, thus increasing the clinical (external) validity. Adherence checks related to concrete interventions are not possible then, or only to a very limited extent. The actual procedure needs to be described, preferably by reports and ratings based on video recordings from the sessions. This has been done repeatedly at our clinic (see for example Grawe et al. [6]) and currently in a study on the integration of emotion-focused therapy elements with an integrative form of cognitive behavioural therapy [7]. This way of providing information on the procedure, reviewing video tapes and describing the actual procedure, is demanding on resources. The definition of experimental conditions by heuristic rules has its price, but it is good that this possibility exists. 
Finally, what is praised as an important contribution to the internal validity of studies, i.e., the selection of a very homogenous group of patients, is also a good trick to increase effect sizes in a situation with necessarily limited differences between the techniques. The smaller the variance in a variable between patients is, the higher the chance to find substantial effect sizes and significant differences. This one also has to keep in mind, when comparing effects reported in RCTs with practice studies or values related to one's own therapies.

To compensate for disadvantages of focusing research on RCTs and techniques, there were several meaningful initiatives, of which the following are particularly worth mentioning:

- the transdiagnostic approach [8] primarily based on the notion that each therapist can learn only a limited number of approaches on a high level of performance

- the APA Division 29 [9], focusing on the impact of the therapeutic relationship on outcome

- the APA Division 12 (Clinical Psychology) and NASPR (North American Chapter of the Society for Psychotherapy) task force, focusing on "crosscutting and integrating principles" [10]: as this approach was very rigorous in the criteria of accepting the principles as evidence, the accepted principles are not comprehensive enough to give a basis for concrete therapies, but the direction of this approach - principles instead of concrete techniques, which actually or supposedly vary from one diagnosis to another - is promising

The very existence of these initiatives further illustrates the limitations of focusing exclusively on RCTs.

\section{Therapeutic Relationship}

The fact that the quality of the therapeutic relationship is positively correlated with therapy outcome is certainly well known to therapists dealing with personality disorders. Even in the absence of a mandate to treat a personality disorder, it needs to be taken into account and dealt with by a therapist treating patients with comorbid personality disorder. The patient brings all requirements for dealing with a particular personality into the relationship with the therapist. The therapeutic relationship can be seen as a microcosm in which a personality disorder unfolds, although it is open to what extent it fully reflects the patient's common way of interacting.

That a positive correlation between the quality of the therapeutic relationship and outcome is probably the most consistent finding in psychotherapy research alto-

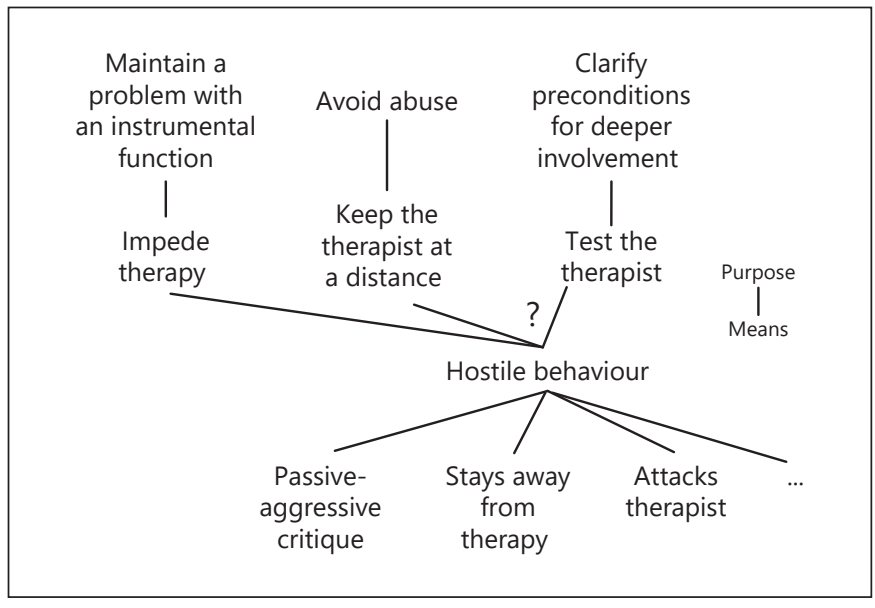

Fig. 1. Searching for an adequate reaction: analysis of possible motives behind hostile patient behaviour.

gether $[11,12]$ does not mean that all depends on this relationship - the effect sizes are only medium - and it even less means that therapists already know what to do for a good relationship based on this finding. As Castonguay [13] once formulated: "How helpful would it be if a supervisor says 'now go and have a good relationship with your patient!'”

Indeed a good relationship is already a micro-outcome in therapy. Therapists are rather in need of information on the ingredients of a good relationship, in particular with difficult patients. They need sufficiently comprehensive and practice-proven prescriptive concepts.

There are not many such concepts. One is the alliance rupture approach [14], with rules such as to engage in non-defensive metacommunication when a rupture occurs. Another approach is the test concept of the Mt. Zion approach [15]. Essentially, it says that patients test their therapist with the unconscious intention of disconfirming pathogenic beliefs. For example, a patient whose selfconcept is to be unlovable behaves in a nasty way to test the therapist really hard whether he/she would still like and engage with him/her. What tests can be expected or identified and how a therapist would have to behave to pass the test is derived from a particular form of case formulation $[16,17]$.

Yet another approach is the complementary, or motive-oriented therapeutic relationship (MOTR) by Grawe and Caspar [18], which is introduced in somewhat greater detail, as it will later also serve as an illustration of a research procedure. It has been developed in its original form in the late 1970s for dealing with "difficult patients" 
at a time when personality disorder diagnoses were not yet popular. Today, many of these patients would satisfy the criteria of having personality disorders.

As its name indicates, the patient's inferred motives determine the right procedure. For example, for patient hostility, there is no trick valid for all of them. Figure 1 illustrates the individualized search for motives to which hostility serves.

When a patient behaves in a hostile way (for example by passive-aggressive critique, staying away from therapy or attacking the therapist openly), we have to ask for the motive hypothetically guiding the behaviour. We may hypothesize that a patient's (probably unconscious) motive is to impede therapy. Why should a patient who comes to therapy voluntarily do this? The motive behind this may be to maintain an instrumental function the problem may have in the patient's life. For another patient, we may hypothesize that she wants to keep the therapist at a distance, as she may have experienced abuse by older male individuals earlier in life, and wants to avoid a repetition of these experiences. Yet another patient may unconsciously want to test the therapist before she or he engages in a deeper phase of therapy. Only if the therapist passes the test with unshaken engagement in spite of the hostility does the patient find him or her sufficiently reliable to take increased risks. This latter hypothetical situation is not based on any problematic therapist behaviour; on the contrary, he or she has done a lot right. Else the patient would not consider going one step further and would not need such a test.

It may be unnecessary to emphasize that no simple concrete trick for how the therapist should react to the respective patient hostility (which may look very similar on the surface) would do justice to the individual situation. MOTR means to support the patient on all levels, but particularly on the level of motives, in the case of a positive, adaptive patient behaviour. The concept shows its specific potency in particular with problematic patient behaviour by the following two principles:

1. to look for an acceptable motive guiding the problem behaviour. Acceptable means that the motive itself (disregarding the problematic behaviour) does not impede the therapist, that is, the therapist could work without major reservations with a patient having this motive. It is guaranteed that going up in the hierarchy of hypothetical motives one will ultimately find such an acceptable motive, as the highest motive corresponds by definition to a human need, which in itself cannot be problematic by nature. One does, however, try to find motives much more concrete than general human needs: the lower the acceptable motive in the instrumental hierarchy, the more specific it is for the individual patient, the easier it can be satisfied, and the fewer therapist resources are needed for it, in contrast to spilling over all potential needs. "As high as necessary, as concrete as possible" is the motto. In the hostility example, the acceptable patient motives may be formulated as "maintain a balance in your functioning," "avoid abuse," and "make sure you are not left out in the rain in a difficult phase"

2. once the unproblematic motive is determined, the therapist tries to satisfy or even oversatisfy it, with the intention to make the problem behaviour superfluous. The patient should not have to use the problematic means when the motive to which it serves is already satisfied. With its motivational base withdrawn, the problem behaviour may disappear very quickly

In our example, the therapist would metaphorically put a ladder to the branch on which the patient sits before an attempt is made to saw it off; he or she would keep a safe distance and use every chance to reassure the patient that a therapeutic relationship and abuse are incompatible, or in the hypothetical test situation keep doing the therapeutic work unshaken by the patient's hostility. It has been shown that following these principles has dramatic positive effects on the process [6] and is also positively correlated to outcome $[19,20]$.

While being in line with the principle of dialectical behaviour therapy [21] of critically discussing problematic (e.g., self-harming) behaviour while valuing the motives behind it, MOTR can be used with any patient, with or without a personality disorder diagnosis. Generally, personality disorders go along with challenges in the therapeutic relationship. Even dependent-submissive and seemingly easy patients are problematic in the sense of pulling the therapist into taking too much responsibility and doing the therapeutic work with the negative side effect of keeping the patient dependent. For schizoid and schizotypal patients, the generally useful concept of therapeutic warmth and closeness may be threatening, as it is for the female patient the father of whom became always especially warm and close preparing a new sexual abuse. MOTR, while using the described two principles, helps to elaborate individual recipes for every patient and is all the more useful, the more problems patients bring into therapy - as common for personality disorder patients. As initially mentioned, the goal here is not to introduce a concept for practice, but, as will follow, for an illustration of possible research.

While it is neither possible nor ethically defendable to run an RCT with half of the therapists introducing a good therapeutic relationship and the other half a bad one, 
add-on designs are possible. The claimed advantage of a prescriptive concept can be tested by providing a relationship as usual to a part of the patients, and a relationship following a particular prescriptive concept to the other half. So far, there have not been many examples for such an experimental procedure, but the existence of, for example, the study by Kramer et al. [20] on the MOTR as an add-on to a psychodynamic treatment of borderline patients shows that such a design is possible.

\section{Therapist Responsiveness}

Responsiveness of therapists to patient characteristics is currently a main topic when it comes to debating how outcome might be optimized. Responsiveness has two meanings in the literature: first, the degree to which a patient is a responder to a particular form of therapy, and second, the degree to which a therapist responds with what he or she offers to the individual needs and possibilities of this patient $[22,23]$. A goal of the latter, the dealing with the requirements of an individual patient for his/her therapy, is to make the impact of the particularities of the patient upon the outcome of therapy disappear. This may seem paradoxical at first, but indeed, in therapy conditions in which the therapists are maximally responsive to patients, much fewer correlations are found between patient pre-therapy variables and outcome $[6,24]$. The lack of a correlation between a variable and outcome does therefore not necessarily mean that the variable is unimportant, but may also indicate that a therapist has taken care of it so well that this variable has lost its impact on outcome. An illustrative example is the variable borderline diagnosis yes versus no. In old times, a yes meant "untreatable." The more specific therapeutic approaches have been developed to successfully treat patients with borderline personality traits or disorder, the less power the borderline variable has on the outcome. In the example above: the better the therapist reacts to hostility, the less the variable patient hostility determines the outcome.

Research which is simply looking for correlation between easy-to-assess variables and outcome may, as it is argued here, lead to completely false conclusions about the importance of a variable. How would research need to be different? The issue here is to assess the extent to which a therapist is responsive. Research would first try to capture the individual situation. This can in most cases only or more easily be reached by means of qualitative analysis. To come to valid, generalizable findings, which must always be the ultimate goal of research, a second, quantifying step is necessary. This will be illustrated with research on the MOTR [19].

The qualitative part consists of deriving a Plan ${ }^{1}$ analysis case conceptualization from video tapes in the beginning of therapy. Plan analysis is a case conceptualization method, which cannot be explained here in any more detail $[18,25]$. It stands here for any kind of case formulation, which includes detailed ideas about the therapeutic relationship. The above analysis of hostility gives an idea how hierarchical Plan structures are inferred, but, of course, a complete analysis is much more comprehensive. The basic unit in such an analysis is the Plan, which includes a purpose or motive, and means to realize it, and in contrast to everyday language, Plans are thought to be largely non-conscious. Plan analysis gives an overview of the instrumental functioning of a patient (bottom up: what are the purposes/motives to which conspicuous behaviours serve?; top down: which means are used to satisfy important motives?).

The quantitative second step consists in a rating of a second, independent set of raters who compare - again based on video tapes - the therapist's intervention with the Plan structure. To what extent is what the therapist does/says complementary to the patient plans? Is he or she supportive in the sense of MOTR or rather blocking/ threatening important Plans? This is rated on a 7-point Likert scale. These values represent a quantitative assessment of therapist responsiveness in the sense of MOTR, which can be used, for example, for correlations with outcome, as in Caspar et al. [19]. The ability of therapists to be responsive is considered to be probably the most important therapist variable contributing to the positive outcome of therapies [3], and it can be expected that responsiveness explains more outcome variance than simple and more-easy-to assess variables, such as therapist directiveness, because the adequacy of different levels of directiveness depends on the patient.

\section{Aptitude Treatment Interaction and Differential Treatment Selection}

The term aptitude treatment interaction (ATI) comes from education research, where it has been found that it depends on the learner, which form of teaching/learning leads to the best results. Also, in psychotherapy it makes

\footnotetext{
1 In the tradition of Miller et al. [36], Plan is written with an uppercase letter emphasizing the difference in meaning (largely non-conscious) compared to everyday language.
} 
sense that "different folks need different strokes" [9, 26]. This may also be behind the repeated finding of equivalent effects whenever bona fide treatments are compared [27]: the mean values of larger groups of patients may disguise existing differences, which are then averaged away. This is in any case one of several explanations for the equivalent paradox; paradox because it is hard to understand why two procedures, which differ in aspects that are expected to be relevant according to convincing concepts, should not lead to different outcomes.

This is the domain of moderator analyses, which, however, often do not lead to significant effects, as they require an even greater number of patients than needed for analyses of mere main effects and are thus often underpowered. However, there are findings, for example that autonomyseeking patients fare better with client-centred therapy, while structure-seeking patients fare better with traditional behaviour therapy [6], or that chronically depressed patients with early traumatization have better outcomes with a cognitive behavioural analysis system of psychotherapy (CBASP) alone than with antidepressant monotherapy, while others do better with a combination of medication and psychotherapy than with psychotherapy alone [28].

Based on this kind of differential findings, the prescriptive psychotherapy approach [30] has been developed and investigated. One would expect that an individualized procedure that takes all variables into account for which moderating effects have been found (for example, the habitual reactance level, internal vs. external coping style, etc.) should lead to a superior outcome in comparison to a standard procedure that does not consider all these variables [26]. The differences were, however, much more limited than one might expect [29]. In general, prescriptive approaches based on the ATI concept had only a moderately positive impact.

How can this be explained? A possible explanation could be that, although ATI means some degree of individualization, focussing on variables is still much above the level of custom tailoring and of individualizing done by experienced practitioners. Although they are more differentiated than common main effect-oriented RCTs, ATI-based RCTs may still lump together groups of patients that are too heterogeneous, and the individualization may not be sufficiently refined.

The solution would then be to individualize much below the level of ATI variables reflecting the practice of good experienced therapists who do not believe in manualization. Indeed it has been shown in other fields of professional expertise that experienced master performers take many details into account and invest into deeper qualitative analysis of a problem before they act $[25,30]$. Does this mean to open the door for an "everything goes" attitude, which one has tried to overcome with evidencebased medicine and psychotherapy? The answer is "no," for several reasons:

- individualization can and has to be based on clear principles, the effects of which can, as argued above, be studied with RCTs;

- when it is difficult to follow a design contrasting individualized therapy, for example focusing on the relationship, with another form of therapy, add-on designs may be possible;

- individualization itself may be assessed as the extent of responsiveness as described above; responsiveness as a variable can then be used and investigated in the process as well as outcome research.

\section{Research on Mechanisms of Change}

There is "a vast number of outcome studies and relatively few studies of mechanisms," as Clarkin and Levy [26] and many others have complained. This lack is severe, as the decision by clinicians whether the results of efficacy studies are applicable to a particular patient [31] requires clear concepts about how therapy and a particular approach to therapy work. There are nearly always differences between a concrete patient and the average of patients in a study, and without such concepts it is hard to guess which of the differences are relevant and to what extent. Most questions practitioners have are related to concrete processes in therapy and working mechanisms rather than to main effects in efficacy studies.

In addition, learning from practice requires, or is at least most precise and efficient, when therapists can relate their observations not only to clear case conceptualizations for the respective patients but also to clear, empirically supported concepts about therapeutic principles and mechanisms of change. It is obvious that we are behind schedule with this kind of research, and that much more resources should flow into it. To add single case analyses to RCT therapies is one promising way to go [32].

\section{Assessment of Patient Variables}

Heterogeneity, ego-syntony, and the resulting ambivalent motivation are particularly important aspects in the assessment in the context of research on psychotherapy for personality disorders. 
Ego-syntony, typical for most personality disorders, limits the validity of self-reports related to all that seems problematic to other persons including significant others, therapists, and raters, but not to the patient. The assessment of therapeutic progress is more difficult than determining whether a previously broken car is running now, or whether or not an anxiety has disappeared. Self-reports reflect the patients' self-concept rather than the "real functioning." To assess the latter, self-report data should be complemented by observational data.

Ambivalent motivation suggests a differentiated assessment of patient motives, stages of change [33], as well as resistance to change, and a concrete therapeutic procedure.

The heterogeneity of all kinds of relevant variables suggests a broad assessment to also capture "non-diagnostic" features, to take care of comorbidity, to consider differential treatment selection [29] and ATI, to think of big samples (possibly implicating multicentric studies with all their challenges and difficulties), as well as the realization and assessment of individualization/responsiveness. The use of goal attainment scaling as a procedure to assess changes based on individually defined goals may also be a good idea.

\section{Making Research on Effects More Precise}

Before coming to a conclusion, I would like to mention a concept that I consider particularly interesting in the light of the heterogeneity of personality disorders: the personalized advantage index as proposed by DeRubeis et al. [34]. They argue, in short, that one should be more specific about expected and examined impacts of the quality of the therapeutic procedures on outcome. At the positive end of whatever variables contribute to making patients easy to treat, it may not make a big difference how good the procedure is. At the other, difficult end, it may make a great difference for individual patients when a brilliant therapist finds an ingenious approach, but on average, even excellent therapists may have such difficulties so that the effect of the quality of their procedure does not become significant. While it is still important to know what the effects as well as mediators and moderators for a larger group are as a whole, it may make sense to concentrate on the malleable part of a sample to study effects and mechanisms. There is evidence that such an approach can be particularly useful [35].

\section{Conclusion}

Overall, it can be stated that generally good, differentiated psychotherapy research is also good for research on psychotherapy for personality disorders. While some aspects are even more important with personality disorders, the wheel does not need to be reinvented.

It has been argued that RCTs have limits which are of particular importance with personality disorders, but that there are feasible adaptations of RCTs as well as alternatives. It has also been argued in favour of an individualization of therapy, based on clear explicit principles and case conceptualization. Moreover, it has been argued that individualized procedures are also amenable to RCTs with appropriate designs, and the extent to which responsiveness is realized in a therapy can also be assessed.

Heterogeneity, ego-syntony, and ambivalent motivation are particularly important in personality disorders, but these phenomena are also known from psychotherapy research in general, and there are ways of dealing with them.

\section{References}

1 Lambert M: The efficacy and effectiveness of psychotherapy; in Lambert MJ (ed): Bergin and Garfield's Handbook of Psychotherapy and Behavior Change, ed 6. New York, Wiley, 2013, pp 169-218.

2 Chambless DL, Hollon SD: Defining empirically supported therapies. J Consult Clin Psychol 1998;66:7-18.

3 Castonguay LG, Hill CE (eds): How and Why Are Some Therapists Better than Others? Understanding Therapist Effects. Washington, American Psychological Association, 2017.
4 Gordon CSS, Jill PP: Parachute use to prevent death and major trauma related to gravitational challenge: systematic review of randomised controlled trials. BMJ 2003;327: 1459-1461.

5 Smith E, Grawe K: Which therapeutic mechanism work when? A step towards the formulation of empirically validated guidelines for therapists' session-t-session decisions. Clin Psychol Psychother 2005;12:112-123.
6 Grawe K, Caspar F, Ambühl HR: Die Berner Therapievergleichsstudie: Fragestellung und Versuchsplan. In Differentielle Psychotherapieforschung: Vier Therapieformen im Vergleich. Z Klin Psychol 1990;4:294-315.

7 Babl A, Grosse Holtforth M, Heer S, Lin M, Stähli A, Holstein D, Belz M, Egenolf Y, Frischknecht E, Ramseyer F, Regli D, Schmied E, Flückiger C, Brodbeck J, Berger T, Caspar F: Psychotherapy integration under scrutiny: investigating the impact of integrating emotion-focused components into a CBT-based approach: a study protocol of a randomized controlled trial. BMC Psychiatry 2016;16:423. 
8 Barlow DH, Farchione TJ, Fairholme CP, Ellard KK, Boisseau CL, Allen LB, May JTE: Unified Protocol for Transdiagnostic Treatment of Emotional Disorders: Therapist Guide. Oxford, University Press, 2010.

9 Norcross J: Empirically supported therapy relationships; in Norcross J (ed): Psychotherapy Relationships That Work. Oxford, Oxford University Press, 2002, pp 3-16.

10 Castonguay L, Beutker L: Principles of Therapeutic Change That Work. Oxford, University Press, 2005

11 Martin DJ, Garske JP, Davis MK: Relation of the therapeutic alliance with outcome and other variables: a meta-analytic review. J Consult Clin Psychol 2000;68:438-450.

12 Flückiger C, Del Re AC, Wampold BE, Symonds D, Horvath AO: How central is the alliance in psychotherapy? A multilevel longitudinal meta-analysis. J Couns Psychol 2012; 59:10-17.

13 Castonguay LG: A common factors approach to psychotherapy training. J Psychother Integr 2000;10:263.

14 Safran JD, Muran JC, Eubanks-Carter C: Repairing alliance ruptures. Psychotherapy (Chic) 2011;48:80-87.

15 Silberschatz G: Testing pathogenic beliefs; in Weiss J, Sampson H; The Mount Zion Psychotherapy Research Group (eds): The Psychoanalytic Process: Theory, Clinical Observation, and Empirical Research. New York, Guilford Press, 1986, pp 256-266.

16 Silberschatz G, Fretter PB, Curtis JT: How do interpretations influence the process of psychotherapy? J Consult Clin Psychol 1986;54: 646-652.

17 Curtis JT, Silberschatz G: Plan formulation method; in Eells TD (ed): Handbook of Psychotherapy Case Formulation. New York, Guilford, 2014, pp 198-220.
18 Caspar F: Plan analysis, ed 2; in Eells TD (ed): Handbook of Psychotherapy Case Formulations. New York, Guilford Press, 2007, pp 221-289.

19 Caspar F, Grossmann C, Unmüssig C, Schramm E: Complementary therapeutic relationship: therapist behavior, interpersonal patterns, and therapeutic effects. Psychother Res 2015;15:91-102.

20 Kramer U, Kolly S, Berthoud L, Keller S, Preisig M, Caspar, F, Berger T, Roten Y, Marquet P, Despland JN: Effects of motive-oriented therapeutic relationship in a ten-session general psychiatric treatment of borderline personality disorder: a randomized controlled trial. Psychother Psychosom 2014;83:176186.

21 Linehan MM, Dialectical behavior therapy for borderline personality disorder: theory and method. Bull Menninger Clin 1987;51:261276.

22 Caspar F, Grosse Holtforth M: Responsiveness - Eine entscheidende Prozessvariable in der Psychotherapie. Z Klin Psychol Psychother 2009;38:61-69.

23 Kramer U, Stiles WB: The responsiveness problem in psychotherapy: a review of proposed solutions. Clin Psychol Sci Pract 2015; 22:277-295.

24 Stiles WB, Honos-Webb L, Surko M: Responsiveness in psychotherapy. Clin Psychol Sci Pract 1998:5:439-458.

25 Caspar F: Beziehungen und Probleme verstehen. Eine Einführung in die psychotherapeutische Plananalyse, ed 4. Bern, Huber, 2018.

26 Clarkin JF, Levy KN: The influence of client variables on psychotherapy; in Lambert MJ (ed): Bergin and Garfield's Handbook of Psychotherapy and Behavior Change, ed 5. New York, Wiley, 2004, pp 194-226.

27 Wampold BE, Imel ZE: The Great Psychotherapy Debate: The Research Evidence for What Works in Psychotherapy. New York, Routledge, 2015.
28 Nemeroff CB, Heim CM, Thase ME, Klein DN, Rush AJ, Schatzberg AF, Ninan PT, McCullough JP, Weiss P, Dunner DL, Rothbaum BO, Kornstein SG, Keitner G, Keller MB: Differential responses to psychotherapy versus pharmacotherapy in the treatment of patients with chronic forms of major depression and childhood trauma. Proc Natl Acad Sci USA 2003;100:14293-14296.

29 Beutler LE, Harwood TM. Prescriptive Psychotherapy: A Practical Guide to Systematic Treatment Selection. Oxford, University Press, 2000.

30 Dreyfus SE: The five-stage model of adult skill acquisition. Bull Sci Technol Soc 2004;24: 177-181.

31 Sackett DL, Strauss SE, Richardson WS, Rosenberg W, Haynes RB: Evidence-Based Medicine: How to Practice and Teach EBM, ed 2. New York, Churchill Livingston, 1996, pp 71-72.

32 Kramer U: Personality, personality disorders, and the process of change. Psychother Res 2017:1-13.

-33 Prochaska JO, DiClemente CC, Norcross JC: In search of how people change: Applications to the addictive behaviors. Am Psychol 1992. 47:1102-1114.

34 DeRubeis RJ, Cohen ZD, Forand, NR, Fournier JC, Gelfand LA, Lorenzo-Luaces L: The Personalized Advantage Index: translating research on prediction into individualized treatment recommendations. A demonstration. PLoS One 2014;9:e83875.

- 35 Forand NR, Huibers MJH, DeRubeis RJ Prognosis moderates the engagement-outcome relationship in unguided cCBT for depression: a proof of concept for the prognosis moderation hypothesis. J Consult Clin Psychol 2017;85:471-483.

36 Miller GA, Galanter E, Pribram KH: Plans and the Structure of Behavior. New York, Holt, Rinehart and Winston, 1960. 